\title{
Uso de macroinvertebrados acuáticos como bioindicadores de la calidad del agua en la cuenca media del rio Guatapurí (Valledupar, Colombia)
}

Julio C. Nuñez y Pedro J. Fragoso-Castilla*

Universidad Popular del Cesar, Grupo de Investigación Parasitología Agroecología Milenio, Valledupar, Cesar - Colombia (correo-e: pedrofragozo@unicesar.edu.co; julio.nunez2901@hotmail.com).

* Autor a quien debe ser dirigida la correspondencia.

Recibido Jul. 17, 2020; Aceptado Sep. 15, 2020; Versión final Oct. 10, 2020, Publicado Dic. 2020

\section{Resumen}

El objetivo del presente estudio fue evaluar la calidad del agua en la cuenca media del Rio Guatapurí (Colombia), a través de análisis de macroinvertebrados acuáticos. Se determinaron los índices de calidad del agua BMWP/Col y ASPT y se estimaron los índices de diversidad alfa. Los 1640 organismos colectados están agrupados en 5 clases, 12 órdenes y 34 familias. La composición de taxones fue diversa y la clase Insecta fue dominante. El orden Hemiptera fue el más diverso y abundante y no se presentaron diferencias en la riqueza ni en la abundancia a nivel espacial. La diversidad alfa presento diferencias significativas entre los diferentes índices ecológicos. Los índices BMWP/Col y ASPT reflejaron una condición de calidad buena, indicando aguas limpias. Se concluye que la cuenca media del Rio Guatapurí se encuentra una buena condición ecológica, después de la valoración y comparación de índices univariados y multivariados.

\section{Use of aquatic macroinvertebrates as bioindicators of water quality in the middle basin of the Guatapurí river (Valledupar, Colombia)}

\begin{abstract}
The objective of the present research study was to evaluate water quality in the middle basin of the Guatapurí River (Colombia) through the analysis of aquatic macroinvertebrates. The BMWP/Col and ASPT water quality indices and the alpha diversity indices were determined. The 1640 collected samples were grouped into 5 classes, 12 orders, and 34 families showing that taxa composition was diverse. The class Insecta was the most dominant. The order Hemiptera was the most diverse and abundant and there were no differences in richness or abundance at the spatial level. There were significant differences in alpha diversity between different ecological indices. The BMWP/Col and ASPT indices were consistent with good quality condition, indicating clean waters. It is concluded that the Guatapurí River middle basin is in good ecological condition, after evaluating and comparing univariate and multivariate indices.
\end{abstract}




\section{INTRODUCCION}

En los ecosistemas acuáticos los macroinvertebrados se han utilizado ampliamente como herramienta para determinar la calidad del agua (Kumar y Khan, 2013; Balderas et al., 2016). Debido a que los macroinvertebrados acuáticos son unos de los organismos acuáticos más empleados para evaluar la calidad del agua puesto que poseen un ciclo de vida más prolongado en comparación con otros organismos acuáticos inferiores y una forma de vida sedentaria, aspectos que se integran como una respuesta a las variaciones espaciales y temporales de las condiciones ambientales (Buss et al., 2015; Carter et al., 2017).

Los ecosistemas acuáticos se destacan por la gran diversidad de especies que pueden albergar (Mori et al., 2017). Sin embargo, los cambios en el uso del suelo de bosques nativos a tierras usadas en agricultura, ganadería y plantaciones forestales, afectan las comunidades acuáticas, ya que la pérdida de vegetación ribereña altera directa e indirectamente la calidad del agua, el tipo de sustrato y afecta las condiciones físicas del hábitat acuático y las comunidades bióticas asociadas (Miserendino et al., 2011; Fierro et al., 2017), disminuyendo el material alóctono que ingresa al cuerpo hídrico y la disponibilidad de sustratos aportados por los bosques adyacentes (Bücker., et al 2010) y alterando la estructura y la función trófica de las comunidades de insectos acuáticos (Lorion y Kennedy, 2009; Iñiguez-Armijos et al., 2018).

En el departamento del Cesar, se han realizado estudios que ofrecen conocimientos sobre las comunidades de macroinvertebrados acuáticos, sobresaliendo los realizados por Rodríguez, (2008), en el cual compararon bentos y los organismos asociados con macrofitas de las ciénagas Zapatosa, Mata de Palma y La Pachita; Martínez-Rodríguez y Pinilla, (2014), evaluaron la calidad del agua de las ciénagas Zapatosa, Mata de Palma y La Pachita; Núñez y Fragoso, (2019), usaron macroinvertebrados acuáticos como bioindicadores en la Ciénaga Mata de Palma asociados a macrofitas y en sedimentos. La mayoría de estos estudios se han realizado en la zona centro del departamento del Cesar, en tanto que la zona norte son pocos los estudios realizados, aunque se destaca la investigación de Madera et al., (2016) en afluentes del Rio Cesar.

El Río Guatapurí está afectado por actividades antrópicas que afectan el recurso hídrico, producto del incremento de turistas que lo frecuentan, además del aumento del volumen de residuos sólidos que llegan al río resultado de todas las actividades humanas; otras fuentes de perturbación son las actividades agropecuarias, la tala de árboles, captaciones para sistemas de riego establecidos en fincas vecinas que puede alterar el cauce de la cuenca, afectando su caudal y la biodiversidad del mismo por alteración del ecosistema. El objetivo del presente estudio fue establecer la calidad del agua en la cuenca media del Rio Guatapurí, mediante la composición y estructura de macroinvertebrados acuáticos, así como definir su estado ecológico, a partir de índices bióticos y ecológicos.

\section{MATERIALES Y METODOS}

Se describe el área de estudio, luego se describen los puntos de muestreo y la recolección de organismos, terminando con una explicación sobre el análisis de variables.

\section{Área de estudio}

El estudio se realizó en la cuenca media del río Guatapurí, en el municipio de Valledupar, al norte del departamento del Cesar (Colombia). El río Guatapurí nace en la cara suroriental de La Sierra Nevada de Santa Marta, a 5.200 m.s.n.m, desde su nacimiento hasta su desembocadura en el río Cesar recorre 78 kilómetros, de los cuales 42 corresponden a la cuenca alta, 22 a la media y 14 a la baja; su cuenca abarca aproximadamente 90 mil hectáreas y está formada por varios ríos, el Donachuí, Curiba, Mamanquea y Los

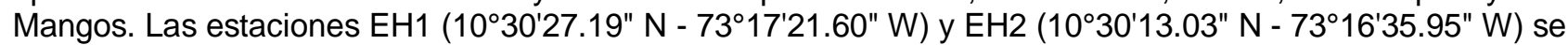
caracterizaron por presentar en su cauce alta turbulencia y remansos además de hojarasca, musgos y algas, su lecho está compuesto por piedras de tamaño grande y mediano, en este sitio hay buena penetración de luz solar; además en este lugar no es común la actividad recreativa. Las estaciones EH3 $\left(10^{\circ} 30^{\prime} 7.00^{\prime \prime} \mathrm{N}\right.$ $\left.73^{\circ} 16^{\prime} 6.81^{\prime \prime} \mathrm{W}\right)$ y EH4 $\left(10^{\circ} 29^{\prime} 46.76^{\prime \prime} \mathrm{N}-7^{\circ} 15^{\prime} 17.01^{\prime \prime} \mathrm{W}\right)$ corresponde al Balneario Hurtado y aguas abajo respectivamente, su cauce presentó tramos de poca turbulencia, mientras que su lecho está constituido principalmente por piedras de menor tamaño, arena y grava; hay actividad antrópica por ser un área turística (Fig. 1).

\section{Fase de campo}

En febrero de 2019, se muestrearon cuatro estaciones en el río Guatapurí, tomando tres replicas por punto de muestreo (4 × $3=12$ muestras). Para la recolección de los macroinvertebrados acuáticos se utilizó la red tipo D-Net, con una luz de malla de $500 \mu \mathrm{m}$. En cada tramo de muestreo se realizó el reconocimiento de los respectivos microhábitats dominantes que fueron sustrato rocoso, hojarasca u orilla con vegetación y 
sedimento fino (arena o gravilla en pozas particular mente), con base en el protocolo propuesto por Álvarez, (2005). Todo el material biológico colectado se preservó y llevó al laboratorio de Microbiología de la Universidad Popular del Cesar de Valledupar para su respectiva separación e identificación taxonómica hasta el nivel de familia con ayuda de claves especializadas (Posada y Roldán, 2003; Álvarez, 2005).

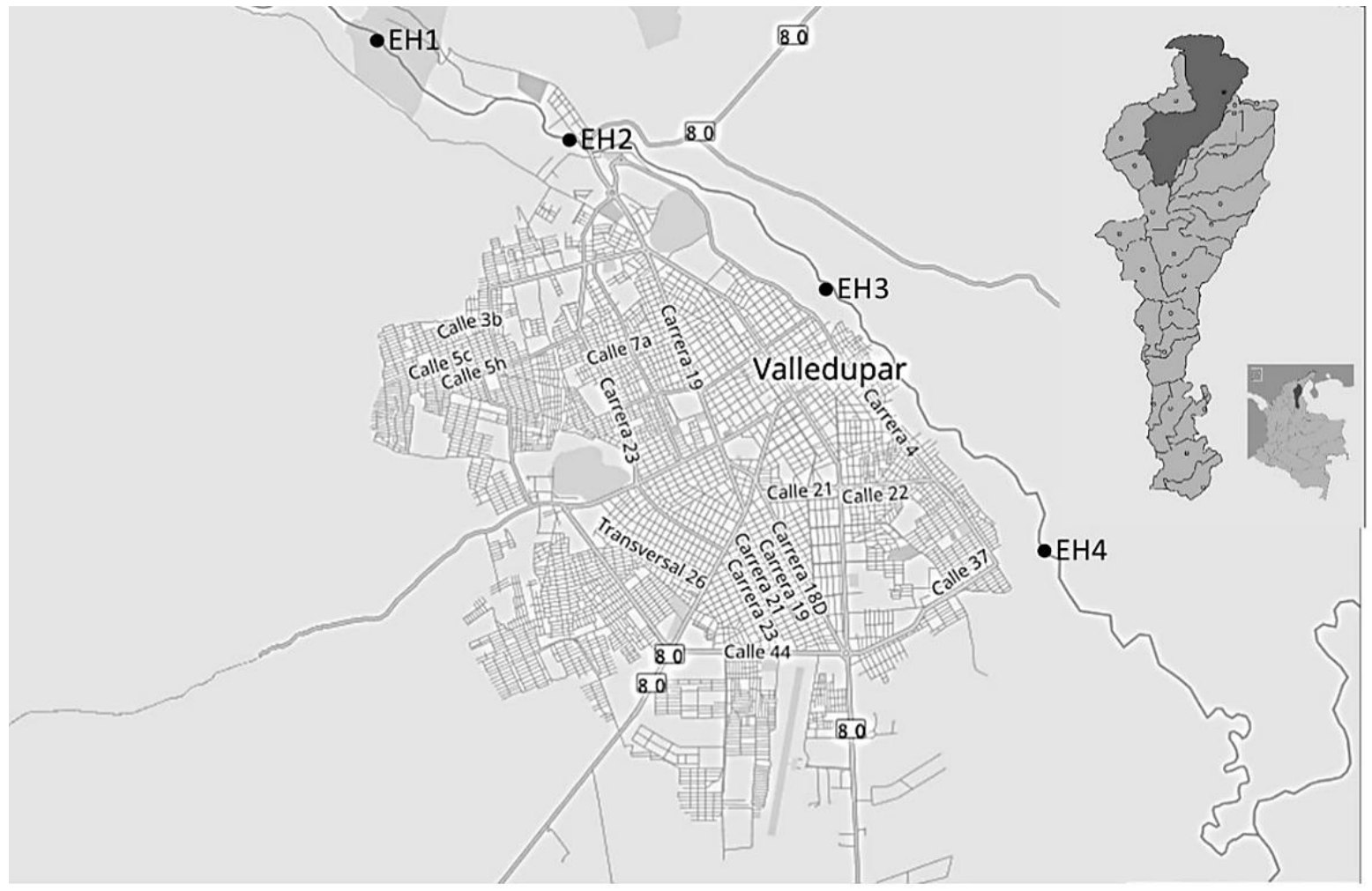

Fig. 1: Ubicación del área de estudio.

\section{Análisis de variables}

Para establecer si existieron diferencias significativas en los valores de riqueza y abundancia, así como de los índices ecológicos alfa entre las estaciones de muestreo (diferencias espaciales), se aplicaron análisis de varianza unidireccionales (ANOVA) a cada variable, previa validación de los supuestos de normalidad (Shapiro-Wilks). Cuando los datos no cumplieron con los supuestos de normalidad se utilizó la prueba no paramétrica de Kruskall-Wallis (K-W) usando el programa estadístico Statgraphics Centurión®, V 16.2.04. La estimación de la riqueza de taxa esperada se analizó con la curva de acumulación de taxa (S), en comparación con la medida del esfuerzo de muestreo (n) usando el programa EstimateS ${ }^{2}$ 9.0. Para el análisis ecológico de algunos atributos estructurales de la comunidad se usaron índices de diversidad alfa de Shannon ' $\mathrm{H}^{\prime}$, dominancia de Simpson D', Uniformidad de Pielou y Riqueza de Margalef' usando el programa Past ${ }^{\circledR} 3.22$. Se calculó el índice biótico BMWP/Col, e índice ASPT (Average Score Per Taxon), para conocer la calidad ecológica del agua.

\section{RESULTADOS Y DISCUSION}

Los resultados se presentan en tres subsecciones: i) caracterización biológica; ii) Índices ecológicos iii) puntaje BMWP/Col y ASPT.

\section{Caracterización Biológica}

En total se recolectaron 1640 organismos agrupados en cinco clases, 12 órdenes y 34 familias (Tabla 1). La clase Insecta tuvo mayor representación en términos de abundancia y riqueza destacándose órdenes como Hemiptera, Ephemeroptera, Trichoptera y Coleoptera (Fig. 3). Aunque la mayoría de los estadios fueron larvales, también aparecieron registros de adultos y pupas. Para este estudio se logró la clasificación de 34 familias, que hace referencia a la riqueza específica global, el ensamble de macroinvertebrados se mantuvo estable, tal como se observa en la curva de acumulación de taxones (Fig. 4). Es probable el registro de nuevos géneros o taxa para esta comunidad, lo que pone de manifiesto la heterogeneidad bioindicadora en el río Guatapurí. El estimador no paramétrico Chao 1 predijo un total de 34 taxones, basado en la abundancia; dicho estimador predice una completitud del inventario del $100 \%$, criterio que define que el muestreo fue representativo. 
Tabla 1. Registro de taxa de macroinvertebrados colectados en cuatro estaciones de muestreo en la cuenca media del río Guatapurí (Valledupar-Colombia).

\begin{tabular}{|c|c|c|c|c|c|c|}
\hline \multirow{2}{*}{ Clase } & \multirow{2}{*}{ Orden } & \multirow{2}{*}{ Familia } & \multicolumn{4}{|c|}{ Estaciones } \\
\hline & & & $\mathrm{EH} 1$ & $\mathrm{EH} 2$ & EH3 & $\mathrm{EH} 4$ \\
\hline \multirow{28}{*}{ Insecta } & \multirow{5}{*}{ Coleoptera } & Elmidae & 65 & 48 & 22 & 34 \\
\hline & & Psephenidae & 12 & 16 & 9 & 8 \\
\hline & & Ptilodactylidae & 17 & 8 & & 6 \\
\hline & & Staphylinidae & 5 & & 7 & \\
\hline & & Lutrochidae & & 2 & & \\
\hline & \multirow{3}{*}{ Ephemeroptera } & Leptophlebiidae & 58 & 87 & 39 & 29 \\
\hline & & Leptohyphidae & 33 & 42 & 22 & 31 \\
\hline & & Baetidae & 22 & 9 & 15 & 18 \\
\hline & \multirow{7}{*}{ Hemiptera } & Gerridae & 123 & 97 & 36 & 21 \\
\hline & & Mesoveliidae & 14 & 34 & & 17 \\
\hline & & Naucoridae & 21 & 15 & 5 & \\
\hline & & Veliidae & 38 & 17 & 12 & 23 \\
\hline & & Hydrometridae & & 4 & & 3 \\
\hline & & Nepidae & & 4 & & \\
\hline & & Corixidae & & & 13 & 18 \\
\hline & \multirow{7}{*}{ Trichoptera } & Glossosomatidae & 11 & 6 & & 10 \\
\hline & & Hydropsychidae & 28 & 34 & 28 & 21 \\
\hline & & Hydroptilidae & 22 & 9 & & 11 \\
\hline & & Leptoceridae & 17 & 11 & 8 & 13 \\
\hline & & Philopotamidae & 11 & & & 22 \\
\hline & & Odontoceridae & 10 & 14 & 16 & 14 \\
\hline & & Calamoceratidae & & 2 & & 6 \\
\hline & Diptera & Chironomidae & 5 & & 7 & \\
\hline & Plecoptera & Perlidae & 18 & 7 & 5 & 8 \\
\hline & \multirow{3}{*}{ Odonata } & Coenagrionidae & 8 & & & 7 \\
\hline & & Libellulidae & & 6 & 7 & 9 \\
\hline & & Gomphidae & 5 & & & \\
\hline & Megaloptera & Corydalidae & & 2 & & 3 \\
\hline \multirow{2}{*}{ Crustacea } & \multirow{2}{*}{ Decapoda } & Pseudothelphusidae & 3 & 2 & & \\
\hline & & Palaemonidae & & 5 & & 4 \\
\hline \multirow{2}{*}{ Gastropoda } & \multirow{2}{*}{ Basommatophora } & Planorbiidae & 4 & 2 & & \\
\hline & & Physidae & 3 & 7 & 3 & \\
\hline Bivalvia & Veneroida & Sphaeriidae & & 3 & & \\
\hline Arachnoidea & Acari & Lynmnessidae & 2 & & & 2 \\
\hline \multicolumn{3}{|c|}{ Numero de organismos colectados } & 555 & 493 & 254 & 338 \\
\hline \multicolumn{3}{|c|}{ Numero de taxas identificados } & 25 & 27 & 17 & 24 \\
\hline
\end{tabular}


Estos registros se relacionan con la heterogeneidad de microhábitats disponibles para la colonización de esta comunidad. Hojarasca, orilla con vegetación, sustrato rocoso y grava en cada tramo muestreado. Los órdenes más abundantes fueron Hemíptera, Ephemeroptera, Trichoptera y Coleóptera con el 31.4 \%, 24.7\%, 19.8\% y $15.8 \%$ de la abundancia absoluta, respectivamente; los demás ordenes reportaron una abundancia absoluta por debajo del $3 \%$. Entre los Hemíptera se presentaron siete familias, siendo las más abundantes Gerridae y Veliidae. Los Ephemeroptera representan tres familias, siendo Leptophlebiidae la más abundante. Trichoptera reporto siete familias, siendo Hydropsychidae la más abundante, mientras que los élmidos fueron el grupo más abundante de los coleópteros. A nivel de familia, Gerridae, Leptophlebiidae, Elmidae, Leptohyphidae y Hydropsychidae (16.9\%, 13\%, 10.3\%, 7.85 y $6.8 \%$, respectivamente) dominaron en todos los puntos de muestreo, además de ser las familias que más morfotipos aportaron.

En cuanto a la abundancia a nivel espacial, EH1 fue la que presento la mayor abundancia de organismos con 555 que representan el $34 \%$ de la abundancia total, en cuanto a diversidad, EH2 fue la que presento una mayor riqueza con 27 familias, mientras que la estación EH3 fue la que reporto menor abundancia con el 15\% y diversidad con 16 familias (Fig 2). Al realizar los análisis estadísticos el nivel de significancia es mayor a 0.05. Esto nos permite aceptar la hipótesis nula $y$, por lo tanto, determinar que no existe una diferencia significativa en la riqueza $(K-W, p=0.55)$ ni en la abundancia $(K-W, p=0.74)$ entre las estaciones evaluadas.
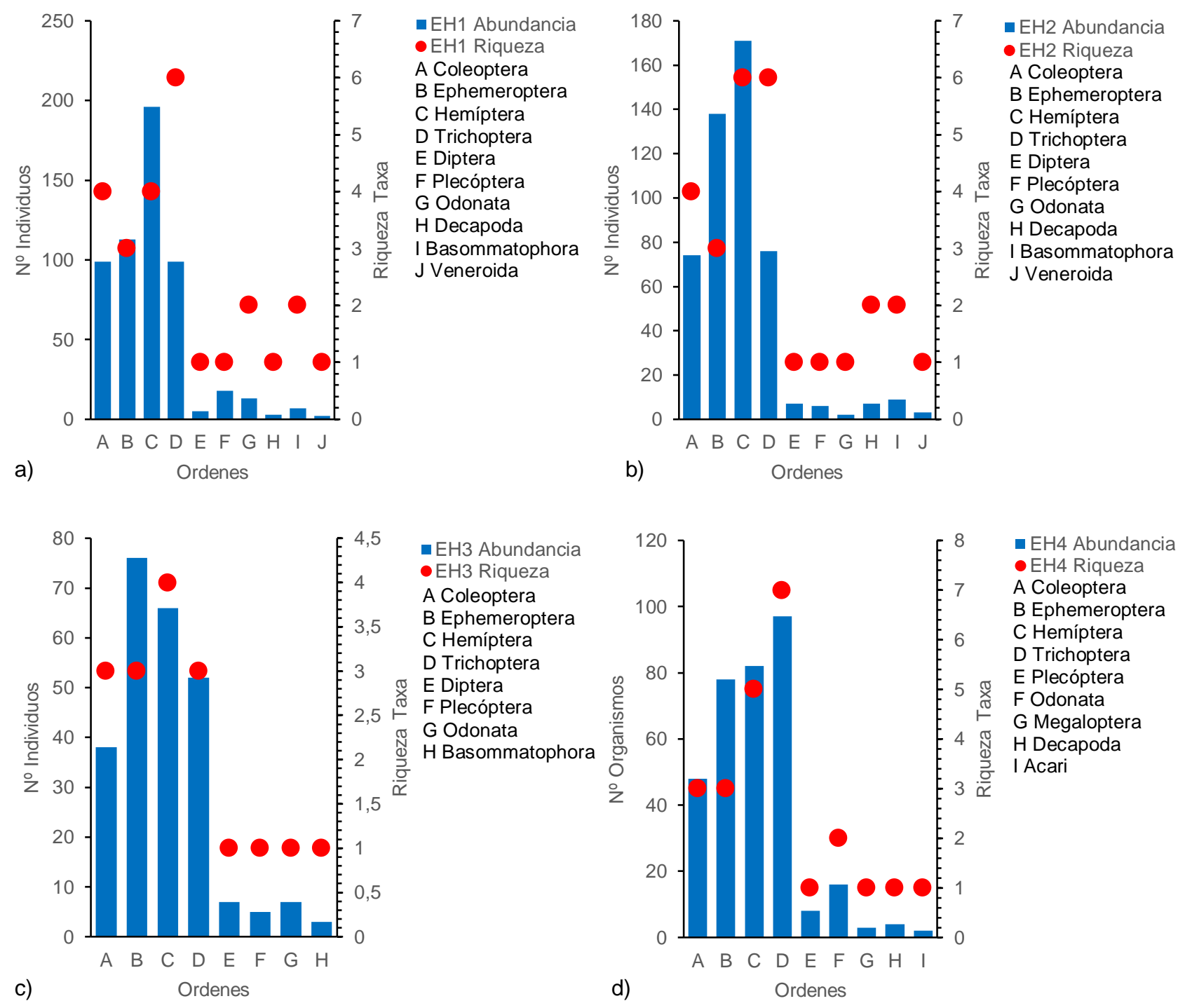

Fig. 2: Distribución de los macroinvertebrados acuáticos en las estaciones de muestreo: A) Estación EH1; B) Estación EH2; C) Estación EH3 y D) Estación EH4.

En cuanto a la riqueza específica global definida por la curva de acumulación de taxa, se acoge la propuesta de Moreno, (2001), quien la considera como un indicador del estado del hábitat, basado en la hipótesis de que un tramo heterogéneo, con alta calidad del agua, permitirá la existencia de un mayor número de taxa (es decir, albergará mayor biodiversidad alfa), que un tramo afectado por alguna acción antropogénica. El orden 
Hemíptera fue el más abundante y el más diverso, de los cuales la familia Gerridae fue la más abundante. Resultados similares fueron reportados por Guinard, Rios y Bernal, (2013) en el rio Gariche provincia de Chiriqui (Panamá) y Nuñez y Fragoso, (2019), en una ciénaga en el centro del departamento del Cesar.

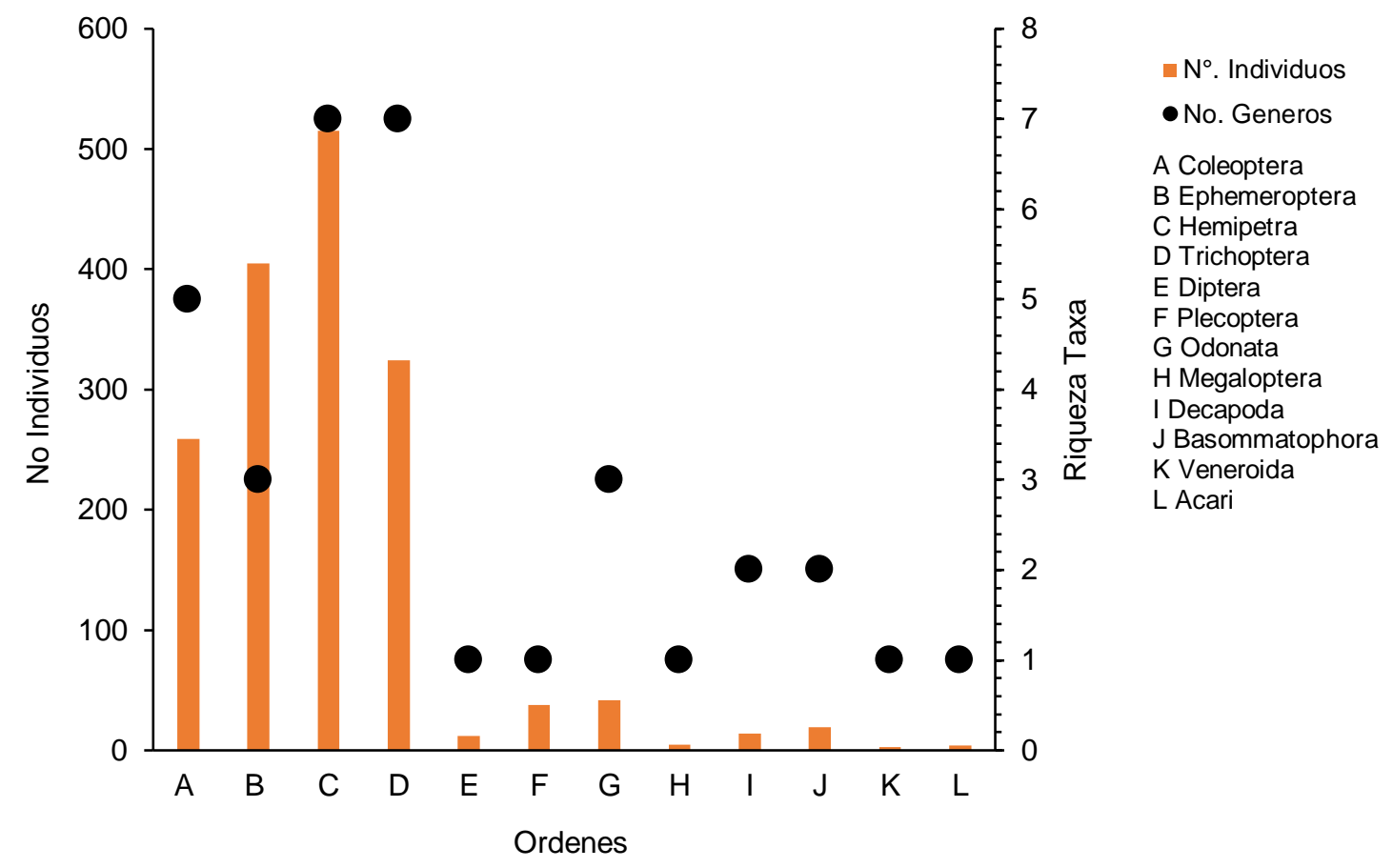

Fig. 3: Composición taxonómica de la comunidad de macroinvertebrados acuáticos en la cuenca media del río Guatapurí (Valledupar-Colombia).

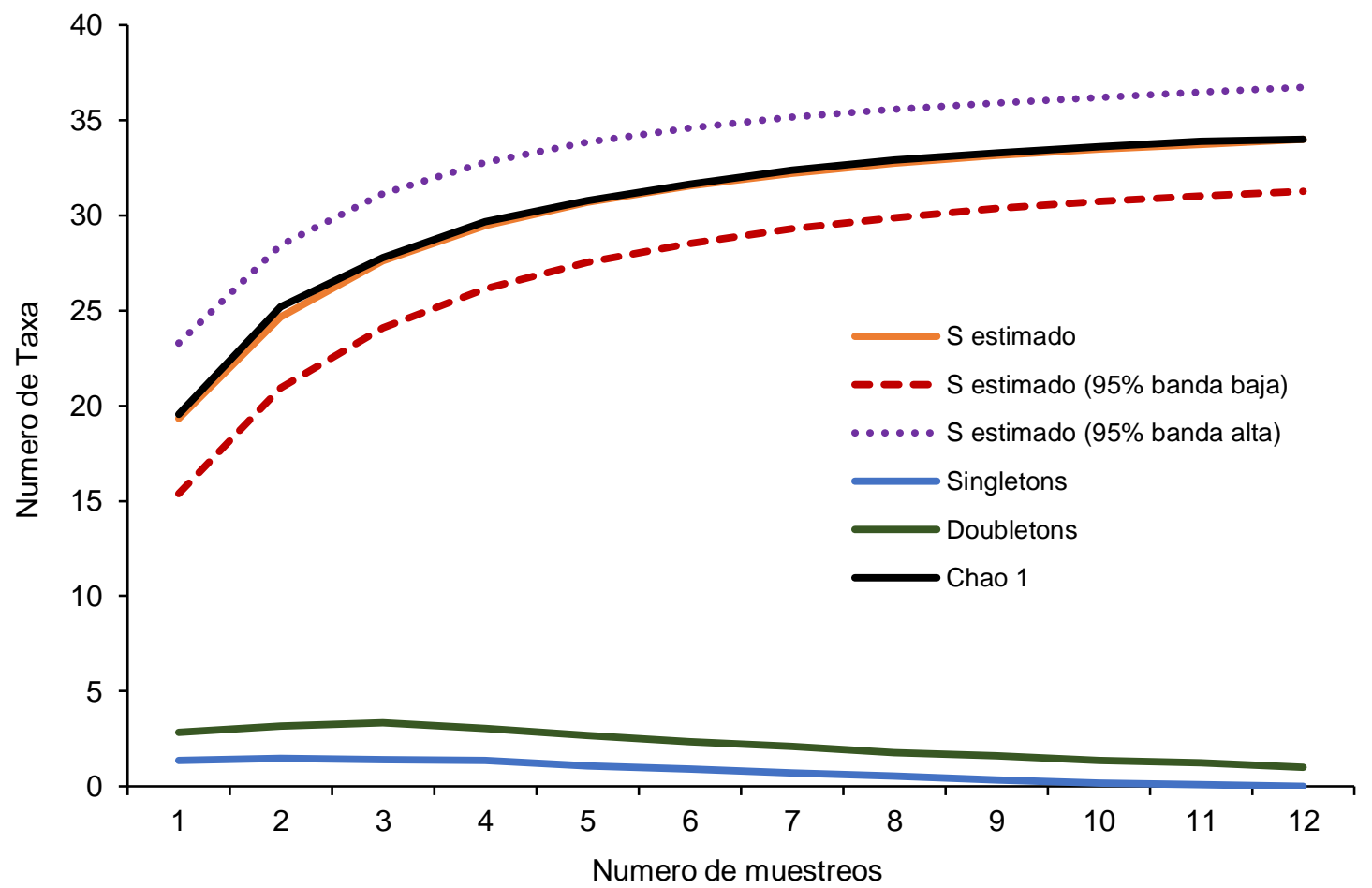

Fig. 4: Curva de acumulación de taxa de los macroinvertebrados acuáticos en la cuenca media del río Guatapurí (Valledupar-Colombia).

El orden Ephemeroptera fue uno de los más importantes y estuvo presente en todas las estaciones de muestreo, aunque solo reporto 3 familias, siendo Leptophlebiidae la más abundante. La diversidad de este grupo es importante en la zona de estudio y resultados similares fueron reportados por Hernández et al., 
(2016) quienes hallaron a los efemerópteros como los más abundantes en una ciénaga y sus afluentes en el centro del departamento del Cesar. Adicionalmente, Madera et al., (2016) en la zona norte del departamento del Cesar reporto tres familias (Leptophlebiidae, Baetidae y Caenidae) en el rio Cesar.

Por su parte, los Tricopteros estuvieron bien representados por larvas y pupas, las cuales viven en diversos ambientes acuáticos y la mayoría construyen refugios fijados al sustrato o refugios portátiles de una variedad de formas y materiales. Estos insectos acuáticos, además de ser diversos, son capaces de colonizar diferentes tipos de sustratos (roca, arena, grava, hojarasca) (Springer, 2008), tanto en rápidos como en remansos. Hydropsychidae fue la familia mas representativa de este orden. El amplio rango de distribución de la familia Hydropsychidae, está asociado a su alta tolerancia a cambios ambientales y su capacidad para construir redes que le permiten fijarse en el sustrato y capturar gran cantidad de alimento; además de ser la familia de mayor distribución y diversidad en Colombia (Vásquez et al., 2014).

Dentro del orden Coleoptera la familia más diversificada en ecosistemas acuáticos es Elmidae. Según (Manzo, 2013), los élmidos son abundantes en la región neotropical, con 46 géneros descritos actualmente. En el presente estudio se encontró una gran diversidad de este grupo, la familia Elmidae fue la más representativa lo que coincide con lo encontrado por Urdanigo et al., (2019). También se destaca la presencia de las familias Ptilodactylidae y Psephenidae las cuales están asociadas a aguas de buena calidad ambiental (O'Callaghan y Kelly-Quinn, 2017)..

Los tramos estudiados del río Guatapurí describen una heterogeneidad espacial, que además de estar influenciada por condiciones biofísicas de la corriente, promueven en la parte alta (EH1 y EH2) un elevado potencial de dominancia tanto de hojarasca como de sustratos rocosos, lo cual influyo en la mayor abundancia y riqueza lo que coincide con lo expresado por Allan y Castillo, (2007) y Walteros-Rodriguez et al., (2016). A nivel de parte baja (EH3 y EH4), existen coberturas importantes de sustrato inorgánico tipo gravas y arenas, pero se mantiene una proporción importante de piedras medianas y pequeñas los cuales aseguran una diversidad funcional en torno a la presencia de organismos, la riqueza y abundancia fue menor en este tramo debido a la actividad antrópica que se presenta por ser un área turística lo que genera un impacto hacia las poblaciones de los organismos acuáticos.

Los resultados de composición y estructura en el ensamble de los macroinvertebrados acuáticos se encuentran soportados por las excelentes condiciones de calidad de hábitat presentes en el río Guatapurí. Algunos órdenes como Coleoptera, Ephemeroptera, Hemiptera y Trichoptera tuvieron buena representación, a nivel espacial (microhábitats). Aunque la mayoría de los estadios fueron larvales, también aparecieron registros de adultos y pupas. Estos resultados fueron similares a los presentados por Walteros-Rodriguez et al., (2016).

\section{Índices Ecológicos}

En cuanto a los estimativos ecológicos evaluados a nivel espacial (Tabla 2), la diversidad Shannon se presentó entre los rangos de 2,60 y 2,87. Por su parte la dominancia de Simpson se encontró entre 0.89 y 0.94, la Riqueza de Margalef oscilo entre 2,89 y 4,19 y la Equidad de Pielou reporto valores entre 0,81 y 0,93. Hubo diferencias estadísticamente significativas entre los diferentes índices ecológicos Shannon $(p=0,016)$; Simpson $(p=0,014)$; Margalef $(p=0,015)$ y Pielou $(\mathrm{K}-\mathrm{W}, p=0,036)$. Existe una riqueza específica y diversidad alfa alta, comparado con estudios en ríos de montaña similares como los presentados por Walteros-Rodriguez et al., (2016).

Tabla 2: Diversidad alfa en cuatro estaciones de muestreo en la cuenca media del río Guatapurí (Valledupar-Colombia).

\begin{tabular}{|l|l|l|l|l|}
\hline \multirow{2}{*}{\multicolumn{1}{|c|}{ Índices Ecológicos }} & \multicolumn{4}{c|}{ Estaciones } \\
\cline { 2 - 5 } & $\mathrm{EH} 1$ & $\mathrm{EH} 2$ & $\mathrm{EH} 3$ & $\mathrm{EH} 4$ \\
\hline Dominancia $(\mathrm{D})$ & 0,90 & 0,89 & 0,91 & 0,94 \\
\hline Equidad de Shannon $\left(\mathrm{H}^{\prime}\right)$ & 2,73 & 2,66 & 2,60 & 2,97 \\
\hline Riqueza de Margalef $(\mathrm{DMg})$ & 3,80 & 4,19 & 2,89 & 3,95 \\
\hline Equidad de Pielou (J') & 0,85 & 0,81 & 0,92 & 0,93 \\
\hline
\end{tabular}

\section{Puntaje BMWP/Col y ASPT}

En primer lugar, el análisis de la calidad ecológica del agua a través de la aplicación del índice BMWP/Col hace referencia a una calidad muy buena, es decir, "agua muy limpia", para cada una de las estaciones con puntajes que van desde 118 a 178 indicando aguas de clase 1 y de buena calidad. Adicionalmente, se 
analizaron los resultados con el índice ASPT donde se obtuvo aguas ligeramente contaminadas, excepto la estación EH1 que reflejo aguas moderadamente contaminadas (Tabla 3), esta diferencia en los resultados se da principalmente porque si bien los valores del índice ASPT depende de los resultados del índice BMWP/Col, se lo considera como un índice más riguroso ya que no solo se basa en la presencia/ausencia de las familias de macroinvertebrados, sino que evalúa también la cantidad de familias encontradas (Daza y Mora, 2016).

Tabla 3. Índices bióticos de calidad de agua en cuatro estaciones de muestreo en la cuenca media del río Guatapurí (Valledupar-Colombia).

\begin{tabular}{|c|c|c|c|c|}
\hline $\begin{array}{l}\text { Estaciones } \\
\text { de muestreo }\end{array}$ & $\begin{array}{l}\text { Puntaje } \\
\text { BMWP/Col }\end{array}$ & Significado & $\begin{array}{l}\text { Puntaje } \\
\text { ASPT }\end{array}$ & Significado \\
\hline $\mathrm{EH} 1$ & 161 & \multirow{4}{*}{$\begin{array}{l}\text { Aguas muy } \\
\text { limpias }\end{array}$} & 6,4 & $\begin{array}{l}\text { Aguas moderadamente } \\
\text { contaminadas }\end{array}$ \\
\hline $\mathrm{EH} 2$ & 178 & & 6,6 & \multirow{3}{*}{$\begin{array}{l}\text { Aguas Ligeramente } \\
\text { contaminadas }\end{array}$} \\
\hline $\mathrm{EH} 3$ & 118 & & 6,9 & \\
\hline $\mathrm{EH} 4$ & 161 & & 6,7 & \\
\hline
\end{tabular}

Las aguas en la cuenca media del río Guatapurí para las estaciones son "muy buenas", ya que el cauce no se encuentra alterado, se reportaron puntajes superiores a 100 indicando aguas muy limpias. Estos resultados son similares a los encontrados por Paulina Ramírez et al., (2020). En el departamento del Cesar se encontraron reportes del uso de este índice en de Madera et al., (2016); Nuñez y Fragoso, (2019). El índice ASPT evidenció aguas entre ligeramente a moderadamente contaminadas a lo largo del tramo estudiado.

\section{CONCLUSIONES}

Los estudios a nivel de campo y laboratorio permiten afirmar lo siguiente: 1) Existe una alta diversidad y composición de macroinvertebrados acuáticos soportados en las excelentes condiciones de los diferentes hábitats monitoreados; 2) El índice BMWP/Col clasifica las aguas del rio Guatapurí en los tramos estudiados como aguas muy limpias; mientras que el índice ASPT clasifica las aguas de ligeramente a moderadamente contaminadas; sin embargo se deben adaptar los índices de calidad como BMWP/Col y ASPT, a la realidad de la misma. 3) Después de la valoración y comparación de índices univariados y multivariados, se confirma una condición ecológica buena para el río Guatapurí en su cuenca media, pero se destaca la necesidad de implementar un monitoreo continuo del cuerpo de agua en su cuenca alta y baja complementado con análisis fisicoquímicos, teniendo siempre presente las estaciones de referencia.

\section{REFERENCIAS}

Allan, D. J., y Castillo, M.M., Stream Ecology: Structure and Function of Running Waters. Second edition. Springer, Dordrecht (The Netherlands). 452 p. (2007)

Álvarez, A., Metodología Para la Utilización de los Macroinvertebrados Acuáticos Como Indicadores de la Calidad del Agua. Instituto de Investigación de Recursos Biológicos Alexander Von Humboldt, N. 05. Bogotá, D.C. (2005)

Balderas, E. C., Grac, C., y otros 2 autores, Potential application of macroinvertebrates indices in bioassessment of Mexican streams, https://doi.org/10.1016/j.ecolind.2015.10.007, Ecological Indicators, 61, 558-567 (2016)

Bücker, A., Sondermann, M., y otros 2 autores. The influence of land-use on macroinvertebrate communities in montane tropical streams a case study from Ecuador, http://dx.doi.org/10.1127/1863-9135/2010/0177-0267, Fundamental and Applied Limnology 177: 267-282. (2010)

Buss, D. F., Carlisle, D., y otros 8 autores, Stream biomonitoring using macroinvertebrates around the globe: a comparison of large-scale programs, http://dx.doi.org/10.1007/s10661-014-4132-8, Environmental monitoring and assessment, 187(1), 4132 (2015)

Carter, J. L., Resh, V., y Hannaford, M., Macroinvertebrates as biotic indicators of environmental quality. https://doi.org/10.1016/B978-0-12-813047-6.00016-4, In Methods in stream ecology (pp. 293-318). Academic Press (2017)

Daza, F., y Mora, C., Evaluación de la calidad de agua de la quebrada el Salitre, utilizando macroinvertebrados acuáticos como bioindicadores para la gestión integral del recurso hídrico (Ingeniería). Universidad Santo Tomás (2016)

Fierro, P., Bertrán, C., y otros 6 autores. Effects of local land-use on riparian vegetation, water quality, and the functional organization of macroinvertebrate assemblages, http://dx.doi.org/ 10.1016/j.scitotenv.2017.07.197, Science of the Total Environment, 609, 724-734 (2017)

Guinard, J., Ríos, T., Bernal, J., Diversidad y abundancia de macroinvertebrados acuáticos y calidad del agua de las cuencas alta y baja del río Gariché, provincia de Chiriqui, Panamá. Revista Gestion y Ambiente 16 (2):61-70 (2013) 
Hernández, J.L., Guzmán-Soto, C.J., y Tamaris-Turizo, C.E., Macroinvertebrados acuáticos de la ciénaga de Sahaya y tres de sus afluentes (Cesar, Colombia). http://dx.doi.org/10.21676/23897864.1857, Revista Intropica 11: 11 - 20 (2016)

Iñiguez-Armijos, C., Hampel, H., y Breuer, L., Land-use effects on structural and functional composition of benthic and leaf-associated macroinvertebrates in four Andean streams, http://dx.doi.org/10.1007/s10452-017-9646-z, Aquatic Ecology 52 (1): 77-92 (2018)

Kumar, P. S., y Khan, A. B., The distribution and diversity of benthic macroinvertebrate fauna in Pondicherry mangroves, India, http://dx.doi.org/10.1186/2046-9063-9-15, Aquatic biosystems, 9(1), 15 (2013)

Lorion, C. M., y Kennedy, B.P., Riparian forest buffers mitigate the effects of deforestation on fish assemblages in tropical headwater streams. http://dx.doi.org/10.1890/08-0050.1, Ecological Applications, 19(2), 468-479 (2009)

Madera, L., Angulo, L., y otros 2 autores. Evaluación de la Calidad del Agua en Algunos Puntos Afluentes del río Cesar (Colombia) utilizando Macroinvertebrados Acuáticos como Bioindicadores de Contaminación, http://doi.org/10.4067/S0718-07642016000400011, Rev. Información Tecnológica 27 (4): 103-110. (2016)

Manzo, V., Los élmidos de la región neotropical (Coleoptera- Byrrhoidea: Elmidae), diversidad y distribución. Revista de la Sociedad Entomológica Argentina 72 (3-4): 199-212 (2013)

Martínez-Rodríguez, M., y Pinillas, G., Assessing the water quality of three wetlands in the Department of Cesar, Colombia, through aquatic macroinvertebrates associated with Eichhornia crassipes (Pontederiaceae), http://dx.doi.org/10.15446/caldasia/v36n2.47489, Caldasia 36 (2): 305-321 (2014)

Miserendino, M. L., Casaux, R y otros 4 autores. Assessing land-use effects on water quality, in-stream habitat, riparian ecosystems and biodiversity in Patagonian northwest streams, http://dx.doi.org/10.1016/j.scitotenv.2010.10.034, Science of the total environment, 409(3), 612-624 (2011)

Moreno, C.E. Métodos para medir la biodiversidad. M\&T-Manuales y Tesis SEA, (1), Zaragoza, 84 p. (2001)

Mori, A. S., Lertzman, K., y otros 2 autores. Biodiversity and ecosystem services in forest ecosystems: a research agenda for applied forest ecology, http://dx.doi.org/10.1111/1365-2664.12669, Journal of Applied Ecology, 54(1), 12-27 (2017)

Núñez, J., y Fragoso, P., Uso de macroinvertebrados acuáticos como bioindicadores de contaminación del agua de la Ciénaga Mata de Palma, Colombia, http://dx.doi.org/10.4067/S0718-07642019000500319, Revista Información Tecnológica 30 (5): 319-330. (2019)

O'Callaghan, P., y Kelly-Quinn, M., Distribution and structure of lotic macroinvertebrate communities and the influence of environmental factors in a tropical cloud forest, Cusuco National Park, Honduras, http://dx.doi.org/10.4081/jlimnol.2016.1522, Journal of Limnology, 76 (1). (2017)

Paulina-Ramírez, Y., Giraldo, L., y otros 3 autores., Influencia de la ganadería en los macroinvertebrados acuáticos en microcuencas de los Andes centrales de Colombia, http://dx.doi.org/10.15517/rbt.v66i3.30316, Revista de Biología Tropical 66 (3): 2018, p (2020)

Posada, J., y Roldán, G., Clave ilustrada y diversidad de las larvas de Trichoptera en el noroccidente de Colombia. Revista Caldasia (Medellín), 21 (1): 169 - 192 (2003)

Rodríguez, M., Macroinvertebrados Bentónicos. En: Estudio de Inventario de Fauna, Flora, Descripción Biofísica y Socioeconómica y Línea Base Ambiental de las Ciénagas de Mata de Palma y La Pachita. Convenio Instituto de Ciencias Naturales/Universidad Nacional de Colombia-Corpocesar. Informe Final de Actividades. pp. 209-238. Bogotá D.C (2008)

Springer, M. Aquatic insect diversity of Costa Rica: state of knowledge. Revista de Biología Tropical, 56(4), 273-295 (2008)

Urdanigo, J. P., Díaz Ponce, M., y otros 6 autores., Diversidad de macroinvertebrados acuáticos en quebradas con diferente cobertura ribereña en el bosque Protector Murocomba, Ecuador. Revista de Biología Tropical, 67 (4): $861-878$ (2019)

Vásquez, J.M., Guevara, G., y Reinoso, G., Factores ambientales asociados con la preferencia de hábitat de larvas de tricopteros en cuencas con bosque seco tropical (Tolima, Colombia). Revista Biología. Tropical 62 (2): 21 - 40 (2014)

Walteros Rodríguez J. M., Castaño Rojas, J.M y Marulanda Gómez, J.H., Ensamble de macroinvertebrados acuáticos y estado ecológico de la microcuenca Dalí-Otún, Departamento de Risaralda, Colombia. Hidrobiológica 26 (3): $359-371$ (2016) 
